\title{
Lógica Difusa para una Descripción de la Gramática de las Lenguas Naturales
}

\author{
Adrià Torrens URrutia* \\ Universitat Rovira i Virgili, Tarragona, Spain
}

\begin{abstract}
Defining the natural language and its gradient phenomena force us to look for formal tools that can represent the bases of a grammar with degrees of grammaticality. The mathematical and formal models are often used in linguistics. And yet, fuzzy logic has not received all the attention it deserves as a tool to explain the natural language processing. Here, we show the theoretical bases that have led us to treat the natural language (NL) inputs gradually. The basis of fuzzy logic for NL are explained here as a tool capable of defining non-discrete values, therefore gradual or fuzzy. A Property Grammar will give the rules of the fuzzy grammar.
\end{abstract}

Keywords: Fuzzy logic, degrees of grammaticality, natural language processing.

Resumen. Definir el lenguaje natural y su gradualidad nos obliga a buscar herramientas formales que puedan definir las bases de una gramática con grados de gramaticalidad. Aunque el uso de modelos matemáticos y formales es habitual en lingüística, la lógica difusa no ha recibido toda la atención que merece como herramienta para explicar el procesamiento del lenguaje natural. Aquí se exponen las bases teóricas que nos han llevado a tratar el lenguaje natural de una manera gradual y las bases de la lógica difusa como herramienta para lidiar con la formalización

\footnotetext{
* Author's address:

Departament de Filologies Romàniques

Universitat Rovira i Virgili

Av. Catalunya 35, 43002 Tarragona, Spain

E-mail adria.torrens@urv.cat
} 
de una gramática que sea capaz de recoger valores no discretos, por lo tanto graduales o difusos. Las reglas de la gramática difusa vendrán dadas por una gramática de propiedades.

Palabras clave: lógica difusa, grados de gramaticalidad, procesamiento del lenguaje natural.

\section{Lingüística y Procesamiento del Lenguaje Natural}

El lenguaje natural se caracteriza por ser un lenguaje inmediato, espontáneo, con errores y ambiguo. Estas características se manifiestan en las producciones lingüísticas como noisy text o texto ruidoso (Baldwin et al. 2013) y suponen un gran inconveniente para el teórico ya que hacen que el análisis del lenguaje sea una tarea extremadamente complicada.

A pesar de que las frases perfectas resultan una excepción en el lenguaje natural, la tendencia en lingüística ha sido establecer unos límites muy claros entre las producciones que son gramaticales y agramaticales: correctas e incorrectas. A este tipo de clasificación binaria la llamamos clasificación discreta o categórica.

Algunos autores han sido firmes defensores de una teoría lingüística completamente categórica que rechace el aspecto gradual del lenguaje, como Joos (1957) y Bouchard (1995). En cambio, otros autores, aunque reconocen que el aspecto gradual es inherente en el lenguaje natural, establecen que la concepción categórica del lenguaje resulta más adecuada a modo metodológico para el estudio del lenguaje natural, como Bever (1975).

La concepción categórica del lenguaje queda reforzada tras la distinción metodológica entre competencia y actuación (Chomsky 1965). Las teorías generativistas están interesadas en generar lenguaje perfecto, sin errores, por lo tanto no les interesa hablar de grados de gramaticalidad, ya que las reglas deben generar ( $\sin$ interferencias) outputs perfectos desde lo más subyacente (competencia) hasta lo más superficial (actuación). Por lo tanto, las tendencias generativistas, a pesar de haber hablado de la existencia de grados de gramaticalidad, conocidos como degrees of grammaticalness (Chomsky 1965: 77), se decantan por la idealización del lenguaje como herramienta metodológica $y$, por consiguiente, obvian o desestiman las tesis que consideran al lenguaje natural como un objeto de naturaleza gradual (Chomsky 1995). 
Sin embargo, lo cierto es que en la mayoría de situaciones entendemos y procesamos frases con agramaticalidades con independencia de la gravedad del error o de la violación de la regla. Si se tiene en cuenta que la capacidad de procesamiento de un input lingüístico es parte de la competencia lingüística, queda claro que la competencia lingüística es capaz de lidiar con una realidad difusa y gradual. Por lo tanto, procesamos el lenguaje de manera gradual (Keller 2000) o en términos difusos (Aarts 2004). Es decir, como humanos, no aceptamos y entendemos solamente las frases perfectas y, por consiguiente, rechazamos las frases con agramaticalidades, sino que nuestra capacidad para procesar las producciones lingüísticas que recibimos (o inputs) es directamente proporcional a su gramaticalidad (o a la cantidad de reglas gramaticales respetadas y violadas).

\section{Gradience: Concepto Lingüístico Clave para la Aplicación de la Lógica Difusa}

Gradience y el concepto de lo difuso en la fuzzy logic comparten un punto teórico principal: ambos buscan clasificar objetos en términos de grado. En gradience el objeto es lingüístico, pues es un concepto que nace en la lingüística. En la lógica difusa un objeto puede ser cualquier elemento en un universo.

\subsection{Gradience y Teorías sobre la Gradación del Lenguaje}

La concepción gradual del lenguaje, o el término gradience, ha aparecido a lo largo de la historia de la lingüística. En Aarts (2004), gradience se define como "un término que sirve para designar el espectro de los fenómenos continuos en el lenguaje, desde las categorías en el nivel de la gramática hasta los sonidos en el nivel de la fonética". De este modo, gradience es un término usado en lingüística para designar fenómenos que, debido a su naturaleza aparentemente difusa, encajan mejor en una explicación no discreta, como por ejemplo, con explicaciones de fenómenos lingüísticos en términos de grado.

Aarts (2004) define dos tipos básicos de gradación para las gramáticas:

- Gradación lineal. La idea es clasificar los objetos lingüísticos en una escala lineal en tres grados. Por lo tanto, en una escala de $[0,1], \alpha$ es $1, \beta$ es 0 y el área media-borrosa es 0.5 . 
- Gradación teórica de conjuntos. Tenemos dos categorías diferentes claramente definidas $(\alpha$ y $\beta$ ). Sin embargo, aquí encontramos gradación cuando ocurre una intersección entre las dos categorías y la definimos como: $\alpha \cap \beta$. En este caso, ambos elementos comparten características que se señalan en la intersección.

A primera vista, ambos tipos de gradación muestran algunos problemas. Por un lado, la gradación lineal es demasiado discreta, ya que solo muestra tres grados. Por lo tanto, esta parece meramente una noción discreta con un tercer grado. Por otro lado, la gradación teórica de conjuntos es demasiado vaga. Simplemente explica las funciones compartidas sin señalar ningún tipo de grado.

Algunos detractores de la gradación usan estas características de gradience para señalar contradicciones. Muñoz (2006) afirma que es contradictorio definir un elemento como vago pero aun así clasificarlo de manera discreta, incluso si esta forma discreta tiene tres grados. Este tipo de críticas con relación a la gradación lingüística son frecuentes. Estas se ven motivadas debido a una mala comprensión de lo que es la gradación lingüística. La visión discreta es gradual en sí misma, pero solo tiene dos grados. La principal diferencia entre el enfoque discreto y el enfoque gradual es la siguiente: el enfoque discreto niega la gradualidad en lingüística mientras que gradience acepta el enfoque discreto y, además, lo mejora para los casos en los que se necesite establecer definiciones para el lenguaje natural jugando con todas las posibilidades de grado entre $1-0$. Donde los enfoques discretos solo ven blanco $(\alpha)$ y negro $(\beta)$, las teorías de gradación lingüística aceptan blanco, negro, y todos los grises posibles entre esos dos.

Las principales teorías que han intentado explicar de un modo formal el aspecto gradual del lenguaje son la Optimality Theory (Prince \& Smolensky 1993), y la Linear Optimality Theory (Keller 2000). Sin embargo, estas teorías son puramente evaluativas y no describen la gramática de una lengua natural.

En este trabajo, partimos de la idea de que el aspecto gradual de una lengua natural (o el fenómeno gradience) y su gramática puede ser explicada mediante dos herramientas: por un lado, el uso de la lógica difusa para definir el marco de una gramática, con Novák (2015) como base teórica y su formalismo Fuzzy Type Theory. Y, por otro lado, el uso de una gramática de restricciones, en este caso, una Gramática de Propiedades (Blache 2016) que 
definirá el conocimiento lingüístico de una lengua así como sus inputs y outputs lingüísticos.

\section{Las Bases Conceptuales de la Lógica Difusa}

La concepción difusa y el concepto de gradience son bastante similares. Sin embargo, es necesario hablar sobre "lo difuso" para una comprensión completa de la noción lingüística de la gradación.

A pesar de que algunos lingüistas como Bolinger (1961) intentaron acercar la concepción de grado a la lingüística, es en matemáticas y en la lógica difusa en donde podemos encontrar enfoques formales serios sobre los fenómenos de grado en relación con muchos incidentes cotidianos, como por ejemplo: la gradación de la temperatura, colores, o expresiones lingüísticas tales como la relación de grado entre alto-bajo, grande-pequeño. Sin embargo, este fenómeno en matemáticas se denomina fenómeno "difuso" y la lógica difusa es la herramienta adecuada para describir formalmente estas relaciones vagas que también se conocen como fuzzy.

Si partimos de un criterio como "altura $=2$ metros $(2$ metros $=1)$ ", la noción discreta diría que una persona solo es alta si alcanza los 2 metros, y las personas que no alcancen esos 2 metros, por ejemplo $1.75 \mathrm{~m}$, siempre son bajas (siempre sería 0). En cambio, la lógica difusa establece que dado un criterio y un índice, por ejemplo 0.75 , en este caso, una persona será más alta cuanto más cercana esté del criterio máximo (1) y será más baja cuanto más lejos se encuentre del criterio máximo.

La lingüística se nutrirá de una mejor comprensión del término gradience a través del vasto campo de trabajo de la lógica difusa. Entendiendo que, dado un criterio de gramaticalidad, un input será más o menos gramatical cuanto más cerca o lejos esté del "estándar dorado" (gold standard) de la lengua natural que estemos trabajando. Por consiguiente, la "norma difusa sientonces" (o fuzzy if-then rule) será la siguiente: si el valor de gramaticalidad es alto, entonces el valor de agramaticalidad será bajo. 


\section{Definición de una Gramática en Términos Difusos}

La Fuzzy Type Theory (Novak 2015) establece la siguiente regla básica para obtener gradación: $A: U \rightarrow L$. Lo que significa que, siguiendo el ejemplo anterior, tenemos una expresión difusa (ser alto) en un set difuso $A$. El set difuso tiene en cuenta elementos $x(208 \mathrm{cms}, 165 \mathrm{cms}, 185 \mathrm{cms})$ en un Universo $U$ (un grupo de gente). El set difuso $A$ "ser alto" asigna a cada elemento $x$ (p.ej. 203cms) en un universo $U$ (un grupo de gente) un grado de verdad o membresía $L$.

En lingüística, se entiende que el set difuso $(A)$ es la Gramática Difusa, el elemento es el input lingüístico en un universo que es las restricciones de una gramática $(U)$. La gramática difusa asigna un grado de pertenencia $L$ a cada uno de esos elementos teniendo en cuenta las restricciones satisfechas y violadas. Las formulaciones fuzzy definen los elementos con grado cuando se usa el tipo omicron en un elemento $\left(M_{o}\right)$.

Siguiendo el formalismo Fuzzy Type Theory formulado por Novák (2015), definimos a una gramática difusa en un sentido multimodal: Gramática Difusa $(\mathrm{FGr}) \equiv^{2}$ fonética $(p h) \&$ morfología $(m) \&$ sintaxis $(x)$ \& semántica $(s) \&$ léxico $(l) \&$ pragmática $(p r) \&$ prosodia $(p s): F G r \equiv(p h, m, x, s, l, p r, p s)$ (en su forma simplificada).

Cada uno de los módulos de la gramática tiene una gramática de propiedades con restricciones lingüísticas que definen el conocimiento lingüístico de cada uno de los módulos. La multimodalidad nos permite ofrecer un grado de gramaticalidad para cada módulo lingüístico al mismo tiempo que un grado de gramaticalidad total, que se dará una vez dada la gramaticalidad de cada uno de los módulos.

Para establecer relaciones de grado en cada elemento lingüístico, el input lingüístico debe ser contrastado con las reglas que definen cada uno de los módulos de la gramática. En este caso, el grado de gramaticalidad uni-modal se conocerá a través del número de reglas satisfechas y violadas en el módulo de una gramática para con un input (por ejemplo, el grado de gramaticalidad en módulo de la sintaxis de una lengua). A continuación presentamos a modo de ejemplo el algoritmo del módulo de la sintaxis de una lengua en una gramática difusa: $\left(X_{(\eta) \gamma} x_{\gamma}\right) d_{\eta}$. Esta fórmula también puede ser expresada de la siguiente manera: $X: X_{\gamma} \times D_{\eta} \rightarrow M_{o}$.

${ }^{2}$ Se lee como "equivalente a". Se denomina equivalencia difusa. Indica que la equivalencia se expresa en términos de grado. 
- $X$ representa el módulo sintáctico de la gramática.

- $X_{\gamma}$ representa el set de las restricciones sintácticas (o reglas) de la gramática.

- $D_{\eta}$ es el set de las restricciones que figuran en un input lingüístico al que llamamos dialecto.

- $M_{o}$ es el set de grados de verdad. Nuestro grado de verdad es equivalente al grado de gramaticalidad.

Por lo tanto, $X$ (que representa a la sintaxis de una gramática) relaciona un conjunto de restricciones sintácticas de una gramática $\left(X_{\gamma}\right)$ con cada restricción del input del dialecto $\left(D_{\eta}\right)$. Cada restricción del conjunto sintáctico de restricciones de una gramática coincidirá con una restricción en un dialecto ( input). Cada restricción coincidente estará vinculada a un grado en $[0,1]$. Dependiendo del número de restricciones satisfechas o violadas, el módulo sintáctico de la gramática dará un valor de grado correspondiente al grado de gramaticalidad de un input lingüístico.

Por lo tanto, con la expresión presentada, los grados de gramaticalidad son calculados sin tener en cuenta ningún tipo de juicio de aceptabilidad por parte de un hablante.

Como hablamos de gramaticalidad y, por lo tanto de competencia, los grados de gramaticalidad no tienen por qué corresponderse con los de aceptabilidad. Por consiguiente, los grados de gramaticalidad son puramente teóri$\cos$ y se establecerá que, por ejemplo, en sintaxis: $X_{\gamma}=\{\gamma \mid \gamma$ el grado de gramaticalidad de una lengua se obtiene de una Gramática de Propiedades; Gramaticalidad Alta (GA): 1-0.8; Gramaticalidad Media (GM): 0.8-0.3; Gramaticalidad Baja (GB): 0.3-0\}.

\section{Conclusiones}

El uso de la lógica difusa y de sus reglas aplicadas a la lingüística con una gramática de propiedades proporciona interesantes descripciones formales que sientan las bases para una gramática que pretenda recoger valores difusos.

Este método consigue explicar fenómenos del lenguaje natural, como por ejemplo los grados gramaticalidad, con una base matemática, así como especificar en dónde se encuentran los grados de gramaticalidad, y, de este modo, 
defender su existencia de un modo formal. De la combinación de lógica difusa y gramáticas de restricción se obtendrá un nuevo método con el que dar cuenta de las variabilidades del lenguaje. Además, las formulaciones lingüísticas con razonamiento lógico-difuso permiten la implementación informática para el desarrollo de nuevas tecnologías con base lingüística.

\section{Bibliografía}

1. Aarts, B. (2004). Conceptions of gradience in the history of linguistics. Language Sciences, 26(4): 343-389.

2. Baldwin, T., Cook, P., Lui, M., MacKinlay, A. \& Wang, L. (2013). How noisy social media text, how different social media sources? In Proceedings of the Sixth International Joint Conference on Natural Language Processing, 1(1): 356-364.

3. Blache, P. (2016). Representing Syntax by Means of Properties: a Formal Framework for Descriptive Approaches. Journal of Language Modelling, 4(2):183-224.

4. Bever, T. (1975). Functional Explanations Require independently motivated fuctional theories. In Grossman, R.E. et al. (eds.), Papers from the Parasession on Functionalism (pp. 580-609). Chicago: Chicago Linguistic Society.

5. Bolinger, D. L. M. (1961). Generality: Gradience and the All-or-none. Berlin: Mouton \& Company.

6. Bouchard, D. (1995). The Semantics of Syntax: A Minimalist Approach to Grammar. Chicago: University of Chicago Press.

7. Chomsky, N. (1965). Aspects of the theory of syntax. Cambridge: MIT Press.

8. Chomsky, N. (1995). The minimalist program (current studies in linguistics 28). Cambridge: MIT Press.

9. Joos, M. (1957). Description of Language Design. Journal of the Acoustical Society of America, 22: 701-708.

10. Keller, F. (2000). Gradience in grammar: experimental and computational aspects of degrees of grammaticality. PhD thesis, University of Edinburgh, Edinburgh.

11. Muñoz, R. (2006). Hacia una ¿nueva? lingüística: reflexiones sobre la llamada alternativa no-discreta. Pandora: revue d'etudes hispaniques, 6(1): 37-58.

12. Novák, V. (2015). Fuzzy Natural Logic: Towards Mathematical Logic of Human Reasoning" In Seising, R., Trillas, E. \& Kacprzyk, J. (eds.), Towards the Future of Fuzzy Logic (pp. 137-165). Berlin: Springer.

13. Prince, A., Smolensky, P. (1993). Optimality Theory: Constraint Interaction in Generative Grammar. Technical Report, Rutgers University, New Brunswick. 


\section{Author's Biodata}

Adrià Torrens Urrutia es doctor en lingüística por la Universitat Rovira i Virgili (Tarragona). Su investigación se sitúa en el área de los lenguajes naturales y formales. Ha trabajado en el desarrollo de las bases formales de una gramática difusa en colaboración con el Laboratoire Parole et Langage en Francia y el Institute for Research and Applications of Fuzzy Modeling en la República Checa. Su investigación se centra en un enfoque interdisciplinario: la combinación de lógica difusa y gramáticas de restricciones para dar cuenta de diferentes fenómenos lingüísticos en términos de grados, tales como grados de gramaticalidad y complejidad. 